\title{
Heterogeneity in Reports of Dementia Disease Duration and Severity: A Review of the Literature
}

\author{
Chiara C. Brück ${ }^{\mathrm{a}, *}$, Frank J. Wolters ${ }^{\mathrm{b}}$, M. Arfan Ikram $^{\mathrm{b}}$ and Inge M.C.M. de Kok ${ }^{\mathrm{a}}$ \\ ${ }^{a}$ Department of Public Health, Erasmus MC, University Medical Center Rotterdam, Rotterdam, the Netherlands \\ ${ }^{\mathrm{b}}$ Department of Epidemiology, Erasmus MC, University Medical Center Rotterdam, Rotterdam, the Netherlands
}

Accepted 16 September 2021

Pre-press 20 October 2021

\begin{abstract}
.
Background: The burden of dementia is changing due to population aging and changes in incidence and risk factor profiles. Reliable projections of future disease burden require accurate estimates of disease duration across different stages of dementia severity.

Objective: To provide an overview of current evidence on severity stage and disease duration in patients with dementia.

Methods: We reviewed the literature on duration of mild cognitive impairment (MCI), dementia, and various dementia severity stages. Data on study setting, country, sample size, severity stages, dementia type, and definition of disease duration was collected. Weighted averages and Q-statistics were calculated within severity stages and duration definitions.

Results: Of 732 screened articles, 15 reported the duration of one or more severity stages and only half of those reported severity stage onset to conversion to the following stage. In those studies, $\mathrm{MCI}$, very mild dementia, and mild dementia stages lasted 3-4 years and moderate and severe dementia stages lasted 1-2 years. Information on the disease duration was reported in $93(13 \%)$ of screened articles and varied from 1 to 17 years. Reporting of dementia severity stage and disease duration in the literature was highly heterogeneous, which was accounted for only in part by dementia type, study setting, or continent of data collection.

Conclusion: The duration of dementia disease stages shortens with advancing stage. However, reliable modelling of future dementia burden and informing of intervention strategies will require more consistently reported duration estimates from studies that follow individuals longitudinally throughout their entire disease course.
\end{abstract}

Keywords: Alzheimer's disease, dementia, disease duration, review, severity, survival

\section{INTRODUCTION}

At present, over 50 million people worldwide are living with dementia, posing a heavy burden on patients, caregivers, and health care systems [1]. Dementia is a progressive neurological disease which, based on symptoms and (in)dependence of the person with dementia, can be divided into three severity stages: mild, moderate, and severe [2]. Fur-

\footnotetext{
${ }^{*}$ Correspondence to: Chiara Brück, Department of Public Health, Erasmus MC, University Medical Center Rotterdam, Dr. Molewaterplein 40, 3015 GD, Rotterdam, the Netherlands. Tel.: +31 010703 8460; E-mail: c.bruck@erasmusmc.nl.
}

thermore, the stage between the cognitive decline of normal aging and the more serious decline of dementia is typically referred to as mild cognitive impairment (MCI) [3]. The evidence on the duration of dementia in the literature is primarily focused on the duration from diagnosis to death. A review on post-diagnosis survival found average survival ranging between 1.1 and 8.5 years depending on a variety of factors such as age, severity at diagnosis, gender, and type of dementia [4]. Another review investigated survival following an Alzheimer's disease or dementia with Lewy bodies diagnosis and found average survival of 5.7 years and 4.1 years, respectively 
[5]. However, evidence on the duration of the different severity stages is fragmented and established estimates are lacking.

Information on the duration of the severity stages is useful for a variety of reasons. First, since the severity stages are directly related to the dependence and care use of the person with dementia [6], duration estimates of the severity stages can help patients and caregivers to plan appropriate services and make necessary arrangements for the future. Second, clinicians and researchers can use information on severity stage durations to get a better insight into the progression and natural history of dementia. Third, policy makers and health care providers are interested in projections of the future dementia burden in light of population aging, changes in risk factor profiles, and disease incidence. Mathematical models are often used to make such projections or evaluate the impact of interventions (i.e., early detection) [7, 8]. Future scenarios can be evaluated in terms of costs and QALYs, but these vary substantially by dementia severity stage $[9$, 10]. Assumptions about the duration of the severity stages are therefore crucial for the accuracy of these projections.

The aim of the study is to provide an overview of current evidence on stage duration, and secondarily on (total) disease duration, in patients with dementia. We therefore reviewed the literature on dementia severity stages (i.e., MCI or very mild dementia, mild dementia, moderate dementia, and severe dementia) and, if stages were not reported, on disease duration.

\section{METHODS}

\section{Search strategy}

We performed a systematized review on contemporary evidence on the durations of dementia severity stages, by searching Medline Ovid and Embase for articles published in the English language including "dementia" as a major term or "dementia" or "Alzheimer" in the title, and in addition, the following terms in either abstract or title: "survival" or "life expectancy" or "staging" or "duration". For detailed information on the search terms, see the Supplementary Material. To limit the impact of changes in diagnostic work-up and incidence over the past decades, we searched for articles published between January 2011 and April 2020.

\section{Study selection}

A total of 2,445 references were found. As duration information is often not mentioned in the abstract, instead of screening the abstract and titles, an automated search of the full text of the 2,445 references for the words "stage", "duration", and "dementia" was performed, which resulted in 732 articles. The full texts of these 732 articles were then screened by a single reviewer (C.C.B.) for numerical quantifications of disease duration. The primary outcome was the duration of severity stages: MCI, very mild dementia, mild dementia, moderate dementia, and severe dementia. If stages were not reported, we extracted (total) dementia disease duration regardless of the severity stage as the secondary outcome.

\section{Exclusion/inclusion criteria}

Results were limited to studies involving human subjects and the following dementia types: Alzheimer's disease (excluding early onset), frontotemporal dementia, dementia with Lewy bodies, vascular dementia, combinations thereof and allcause dementia. Reviews, meta-analyses, conference or poster abstracts, letters, notes, authors responses, or editorials were excluded.

\section{Data extraction and analyses}

For the primary analysis, stage duration estimates for the beforementioned stages were extracted and for the secondary analysis, disease duration estimates were extracted. For all articles, data on the setting (population-based or clinical), country of data collection, dementia type, sample size, and definition of disease duration was extracted. The collected definitions were then grouped into six post-hoc defined categories: (a) severity stage onset to conversion to the following stage, (b) overall disease onset to study entry, (c) overall disease onset to diagnosis, (d) diagnosis to death, (e) overall disease onset to death, and (f) study entry to death. "Overall disease onset" refers to onset of symptoms according to the patient, the caregiver or a clinician's indication. "Severity stage onset" refers to time of conversion from the preceding stage. Study entry is defined as the baseline study assessment. The analysis was performed separately for the primary outcome (severity stage durations) and the secondary outcome (disease duration). Within the severity stages (only for primary analysis) and definition categories, we calculated weighted aver- 


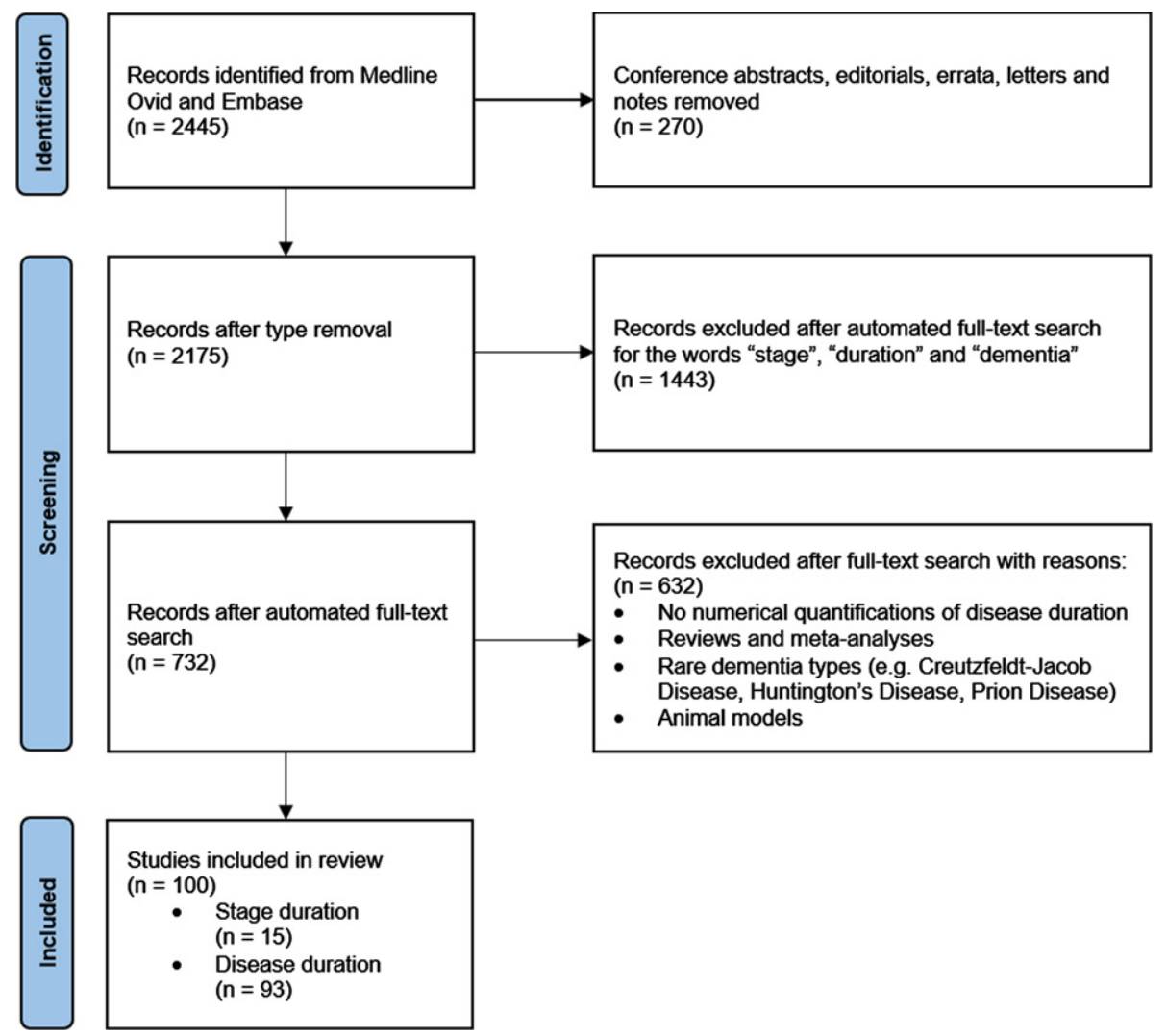

Fig. 1. Flowchart of identification, screening, and inclusion of studies.

ages by sample size and Q-statistics (calculated as the, by sample size weighted, sum of squared differences between individual study estimates and the weighted average) [11].

\section{RESULTS}

\section{Severity stage durations}

Of 732 screened articles, 15 reported the duration of one or more severity stages. Most articles reported estimates based on clinical data $(13 / 15$; $87 \%$ ), whereas one article reported population-based estimates and another reported both clinical and population-based estimates. Clinical duration estimates were on average higher than population-based estimates (Supplementary Table 1). Eleven articles focused on Alzheimer's disease, two on all-cause dementia, one on frontotemporal dementia and one on dementia with Lewy bodies. Duration estimates for Alzheimer's disease were on average higher than for mixed dementia or dementia with Lewy bodies. The data used in the articles originated mostly from
Europe $(8 / 15 ; 53 \%)$, followed by North America $(4 / 15 ; 27 \%)$ and Asia $(3 / 15 ; 20 \%)$. American duration estimates were on average higher than European estimates.

The definition of stage duration varied (Fig. 2), and only half of the studies reported the duration from onset of one stage to conversion to the next. Of the articles reporting stage specific durations (i.e., severity stage onset to progression), two articles reported estimates for all severity stages and six reported estimates only for the $\mathrm{MCI} /$ very mild dementia stage (Fig. 2A). The MCI/very mild dementia stage lasted on average 3.1 years, the mild dementia stage 3.5 years, the moderate dementia stage 2.0 years, and severe dementia stage 1.3 years (Table 1). The variability was higher in the MCI stage $(Q$-statistic $=2.29)$ than in any of the other stages $(\mathrm{Q}$-statistic $=0.39-0.64)$. This variability decreased considerably when stratifying the analysis by dementia type, study setting (clinical or population-based) and continent of data collection (Supplementary Table 1). The variability in the MCI stage was higher in population-based than in clinical 

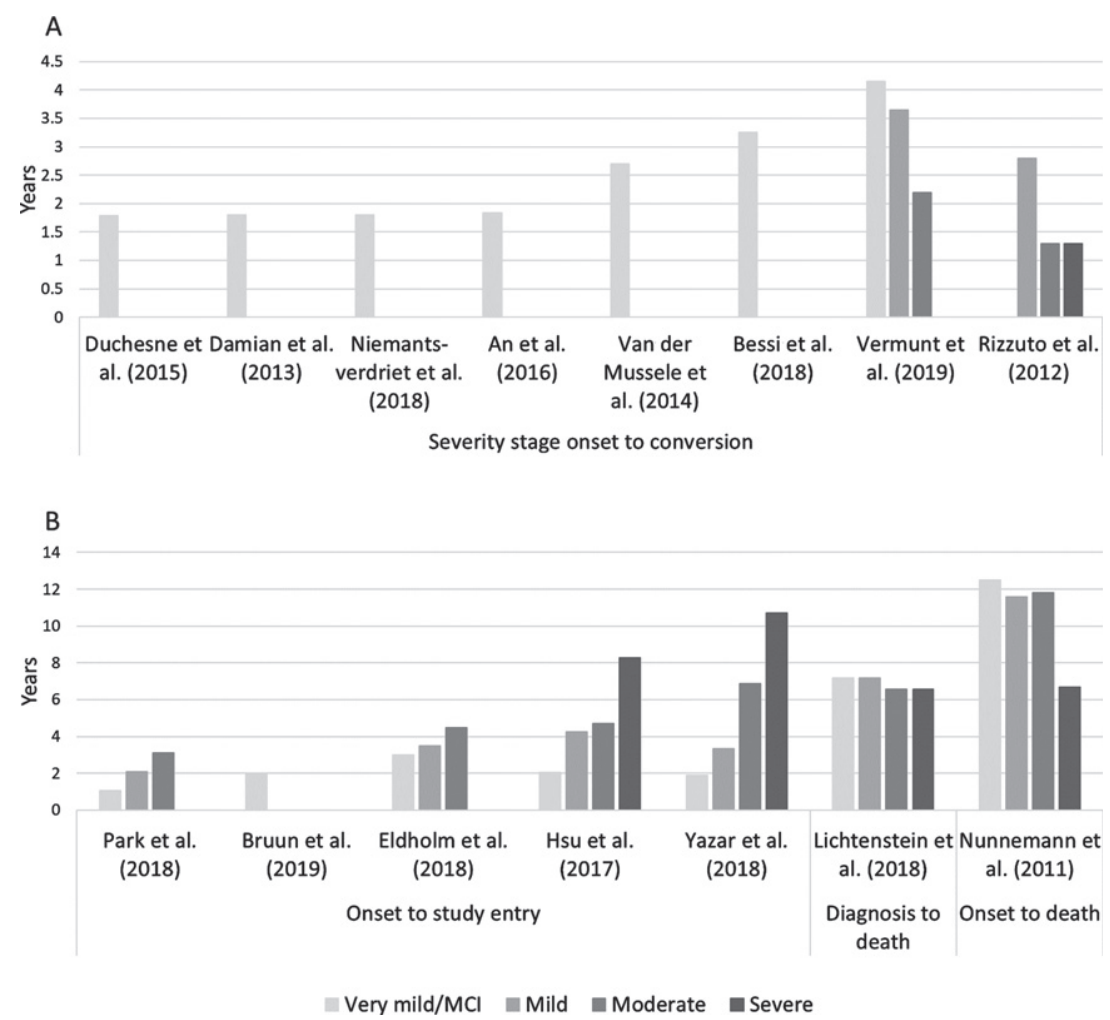

Fig. 2. Severity stage duration estimates in years by duration definition.

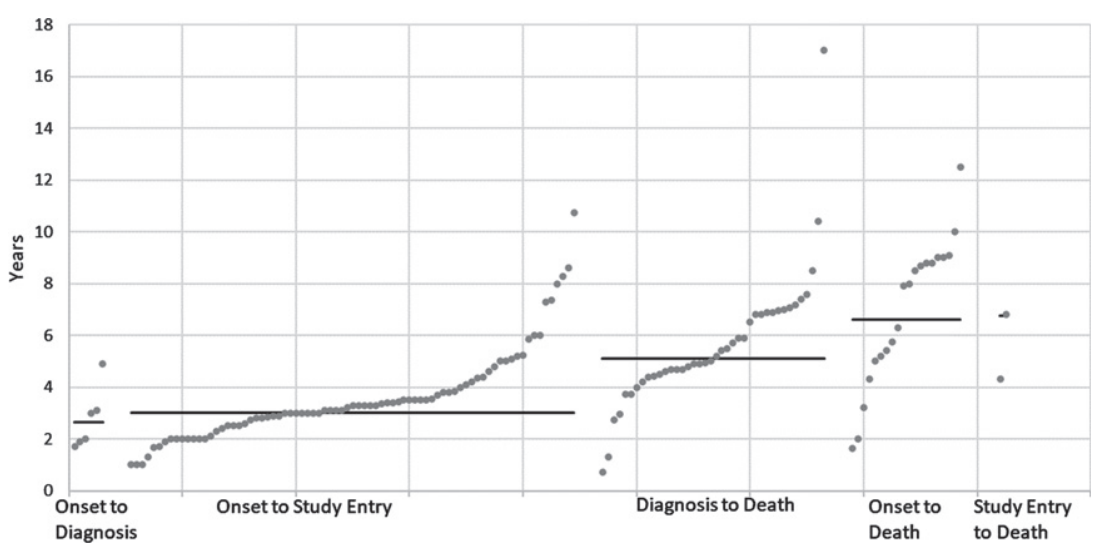

Fig. 3. Total disease duration estimates in years (grey dots) with weighted average (black line, weighed by sample size) by duration definition.

studies, and higher in studies from Europe than North America (Supplementary Table 1).

For articles reporting on duration from overall disease onset to study entry, the stage durations naturally increased with severity (Fig. 2B), as onset was defined as the onset of dementia instead of the onset of a particular severity stage. The variability was generally lower among these studies than amongst the studies reporting stage specific durations.

\section{Disease duration}

In our search for severity stage durations, we also identified ninety-three articles that reported disease duration, irrespective of the severity stage. These 93 articles included 144 estimates of disease duration, as some articles reported different durations by age, sex, or dementia type. Most of the articles based their estimates on clinical data (75/93; 
Table 1

Severity stage duration and total disease duration estimates and Q-statistic

\begin{tabular}{lcccc}
\hline & $\begin{array}{c}\text { Articles } \\
\text { (n) }\end{array}$ & $\begin{array}{c}\text { Estimates } \\
(\mathrm{n})\end{array}$ & $\begin{array}{c}\text { Stage duration } \\
\text { (weighted } \\
\text { average, y) }\end{array}$ & Q-statistic \\
\hline Severity Stage Duration & 15 & 42 & & \\
Severity stage onset to conversion & 8 & 21 & 3.11 & 2.29 \\
MCI/very mild dementia & 8 & 11 & 3.32 & 0.39 \\
Mild & 2 & 4 & 1.99 & 0.42 \\
Moderate & 2 & 4 & 1.30 & 0.64 \\
Severe & 1 & 2 & & 0.25 \\
Overall disease onset to study entry & 5 & 15 & 2.27 & 0.89 \\
MCI/very mild dementia & 5 & 5 & 2.96 & 0.83 \\
Mild & 4 & 4 & 4.90 & 1.19 \\
Moderate & 4 & 4 & 8.94 & - \\
Severe & 2 & 2 & 7.17 & - \\
Diagnosis to death & 1 & 2 & 6.58 & - \\
MCI/very mild or mild dementia & 1 & 1 & & - \\
Moderate to severe & 1 & 1 & 12.5 & - \\
Overall disease onset to death & 1 & 4 & 11.6 & - \\
MCI/very mild dementia & 1 & 1 & 11.8 & 1.32 \\
Mild & 1 & 1 & 6.7 & 2.19 \\
Moderate & 1 & 1 & & 3.53 \\
Severe & 1 & 1 & 2.63 & 0.06 \\
Disease Duration & 93 & 144 & 3.03 & 5.12 \\
Overall disease onset to diagnosis & 5 & 6 & 6.77 & \\
Overall disease onset to study entry & 58 & 78 & & \\
Diagnosis to death & 14 & 38 & 20 & \\
Overall disease onset to death & 14 & 2 & & \\
Study Entry to death & 2 & & & \\
\hline
\end{tabular}

$80 \%$ ), whereas 18 (20\%) used population-based data. Again, clinical duration estimates were on average higher than population-based estimates (Supplementary Table 1). The articles used data from Europe (45/93), the United States and Canada (36/93), Asia (12/93), Australia (2/93), and South America (1/93). Estimates from Asia were on average higher than those from North America or Europe.

Disease duration estimates ranged from 1 to 17 years. However, the definition of disease duration was heterogeneous and could refer to every possible section of the natural history (i.e., overall disease onset to study entry, diagnosis to death, overall disease onset to death) (Fig. 3). The average time between overall disease onset and diagnosis was 2.6 years, the average time from diagnosis to death 5.1 years, and the average time from overall disease onset to death 6.6 years (Table 1). The variability was highest in overall disease onset to death $(\mathrm{Q}$-statistic $=3.69)$ and overall disease onset to study entry $(\mathrm{Q}$-statistic $=2.19)$.

On the estimate level, different types of dementia were studied: 74 estimates for Alzheimer's disease, 16 for dementia with Lewy bodies, 11 for frontotemporal dementia, 3 for vascular dementia, and 40 for a study population with mixed or all-cause dementia. Across the different duration categories, no clear pattern between average duration and dementia types was visible.

When stratifying by dementia type, study setting and continent of data collection, the variability decreased in most cases (Supplementary Table 1). Higher variability was found in studies from Asia, and, in some duration categories, for studies about dementia with Lewy bodies and frontotemporal dementia.

\section{DISCUSSION}

Despite their importance for policy and care planning, information on the duration of the severity stages of dementia is scarce on the basis of this review. We identified fifteen articles mentioning severity stage durations, of which eight provided estimates from the severity stage onset to progression and only two reported severity stage durations within individuals across more than one stage. Severity stage durations decreased with severity, with MCI or very mild dementia and mild dementia stages lasting 34 years and moderate and severe dementia stages lasting 1-2 years.

Mathematical models that simulate the development and progression of dementia in a population 
require accurate duration information to inform the natural history structure of the model. For evaluations in terms of health care use, costs and QALYs, which vary substantially by severity $[9,10]$, the stratification by different severity stages is of great importance. There are two types of simulation models that require different duration information. First, state-transition models with fixed cycle lengths (i.e., one year) require transition probabilities that describe the changes between stages at the end of a cycle (i.e., [12-15]). Second, models that simulate dementia on a continuous time scale require duration estimates that are often calibrated based on trial or observational data (i.e., [16-18]). The estimates presented in this paper can be used as input for the second model type without the need of parameter calibration. Accurate duration estimates are crucial for the estimation of model output. Given a certain incidence, assuming durations that are too short will result in an underestimation of important measures such as the prevalence of dementia, costs or QALYs lost. Similarly, too long durations will result in an overestimation. Therefore, models that project the future burden of dementia or evaluate the potential effects of interventions require accurate estimates of dementia severity stage duration to avoid an under- or overestimation of, for example, dementia prevalence or the effect of an intervention. Additional reports of severity stage durations spanning the entire disease course are needed to facilitate development of consistent models predicting the future burden of dementia.

The greatest challenge in providing accurate estimates of severity stages, is the heterogeneity in reported estimates in literature. The term "disease duration" can refer to every possible section of the natural history (i.e., overall disease onset to death, diagnosis to death) as well as study design specific measures (i.e., overall disease onset to study entry). Measures with study design specific start or end points provide limited information regarding total disease duration as study entry may occur at any point in the progression of the disease. Furthermore, details on the definition of disease duration are in almost half of the articles not mentioned explicitly $(46 / 100 ; 46 \%)$. This lack of transparency and wide range of possible definitions are a clear source of the heterogeneity and ambiguity in reporting of disease duration. Reporting of the start (i.e., overall disease onset, study entry or diagnosis) and the end point (i.e., study entry, diagnosis, or death) of the duration measure can help to avoid ambiguity. Consistent terminology, with clear definitions of terms such as disease duration or survival, can facilitate comparison and meta-analyses of reported duration estimates. As advocated previously [4], we therefore recommend 1) reporting the definition of disease duration explicitly; and 2) using consistent terminology.

Furthermore, the estimates of dementia disease duration within the same duration category vary substantially, due in part to the heterogeneity of dementia, as a syndrome diagnosis, and the inclusion of various study populations in terms of dementia type, study setting and location. In addition, there are a number of methods to assess the severity of dementia (i.e., CDR, MMSE) which are often highly correlated $[19,20]$ but can give different results, thus influencing the comparability of studies using different methods. The heterogeneity in the severity stage duration estimates might therefore be partly due to the use of different severity assessments (Supplementary Table 2). Even with consistent diagnostic criteria, dementia remains a syndrome diagnosis without biological measurements or imaging markers [2], inevitably introducing heterogeneity. Most articles report duration estimates based on clinical data, collected for example in memory clinics or hospitals. Patients included in clinical studies do not necessarily reflect their community-dwelling counterparts, and limited follow-up may prohibit accurate estimation of the entire disease course of study participants. These limitations could be alleviated by following individuals longitudinally throughout the disease course in clinical studies as well as unselected populations.

Some limitations to our analysis should be taken into account. First, severity stage duration estimates are often not the main topic of an article, hampering a truly systematic search of the literature. Nevertheless, by performing a thorough full-text rather than title/abstract search, we expect to have identified most of the relevant reports on stage duration. In contrast, we did not intend to provide a complete literature overview of (total) dementia disease durations, as these were derived only from studies that were identified within the main search on stage severity. This may have biased total disease durations, if for instance clinical studies report more often on disease duration than population-based studies. Nevertheless, the post-diagnosis survival estimates of this study compare well with those of the beforementioned systematic reviews: this study finds an overall average of 5.12 years, 6.30 years for Alzheimer's disease and 4.00 years for dementia with Lewy bodies, Brodaty et al. [4] found a range of 1.1 to 8.5 years, and Mueller et al. [5] found an average of 5.7 years 
for Alzheimer's disease and 4.1 years for dementia with Lewy bodies. Although the information gathered on (total) disease duration might not be complete, we believe the here presented results remain highly informative, especially regarding the heterogeneity of the disease duration definition. Third, there was substantial heterogeneity in duration estimates between studies. Identifying sources of heterogeneity in future studies may benefit reliability of patient information and generalizability of prediction models. Fourth, the identified studies were published within the last 10 years, and when extrapolating these findings to future dementia risk it is important to consider potential changes in stage durations, due to for instance changes in diagnostic practices or compression of morbidity [21].

As a conclusion, time durations of dementia severity stages are scarcely reported in literature, and differences in included populations and duration definition hamper comparability. On average we found that severity stage durations decrease with severity, with MCI or very mild dementia and mild dementia stages lasting 3-4 years and moderate and severe dementia stages lasting 1-2 years. Accurate descriptions of the natural history of disease are important for care planning for individual patients, as well as mathematical models that predict the future burden of dementia and evaluate effects of interventions in healthcare systems. To enable reliable modelling of dementia disease durations in the population, there is need for consistently reported estimates from studies that follow individuals longitudinally throughout their disease course.

\section{ACKNOWLEDGMENTS}

The authors wish to thank Wichor M. Bramer, PhD from the Erasmus MC Medical Library for developing and updating the search strategy.

This work was supported by a VENI grant from the Dutch Research Council (grant number 016.Veni.198.020).

Authors' disclosures available online (https:// www.j-alz.com/manuscript-disclosures/21-0544r2).

\section{SUPPLEMENTARY MATERIAL}

The supplementary material is available in the electronic version of this article: https:// dx.doi.org/10.3233/JAD-210544.

\section{REFERENCES}

[1] Patterson C (2018) World Alzheimer Report 2018. The state of the art of dementia research: New frontiers. Alzheimer's Disease International, London.

[2] American Psychiatric Association (2013) Neurocognitive disorders. In Diagnostic and Statistical Manual of Mental Disorders, Washington, DC.

[3] Gauthier S, Reisberg B, Zaudig M, Petersen RC, Ritchie K, Broich K, Belleville S, Brodaty H, Bennett D, Chertkow $\mathrm{H}$, Cummings JL, de Leon M, Feldman H, Ganguli M, Hampel H, Scheltens P, Tierney MC, Whitehouse P, Winblad B (2006) Mild cognitive impairment. Lancet 367, 1262-1270.

[4] Brodaty H, Seeher K, Gibson L (2012) Dementia time to death: A systematic literature review on survival time and years of life lost in people with dementia. Int Psychogeriatr 24, 1034-1045.

[5] Mueller C, Soysal P, Rongve A, Isik AT, Thompson T, Maggi S, Smith L, Basso C, Stewart R, Ballard C (2019) Survival time and differences between dementia with Lewy bodies and Alzheimer's disease following diagnosis: A meta-analysis of longitudinal studies. Ageing Res Rev 50, 72-80.

[6] Rattinger GB, Schwartz S, Mullins CD, Corcoran C, Zuckerman IH, Sanders C, Norton MC, Fauth EB, Leoutsakos JM, Lyketsos CG, Tschanz JT (2015) Dementia severity and the longitudinal costs of informal care in the Cache County population. Alzheimers Dement 11, 946-954.

[7] Norton S, Matthews FE, Brayne C (2013) A commentary on studies presenting projections of the future prevalence of dementia. BMC Public Health 13, 1-5.

[8] Kretzschmar M (2020) Disease modeling for public health: Added value, challenges, and institutional constraints. $J$ Public Health Policy 41, 39-51.

[9] Cantarero-Prieto D, Leon PL, Blazquez-Fernandez C, Juan PS, Cobo CS (2020) The economic cost of dementia: A systematic review. Dementia (London) 19, 2637-2657.

[10] Martyr A, Nelis SM, Quinn C, Wu Y-T, Lamont RA, Henderson C, Clarke R, Hindle JV, Thom JM, Jones IR (2018) Living well with dementia: A systematic review and correlational meta-analysis of factors associated with quality of life, well-being and life satisfaction in people with dementia. Psychol Med 48, 2130-2139.

[11] Cochran WG (1954) The combination of estimates from different experiments. Biometrics 10, 101-129.

[12] Hadjichrysanthou C, Ower AK, de Wolf F, Anderson RM, Initiative AsDN (2018) The development of a stochastic mathematical model of Alzheimer's disease to help improve the design of clinical trials of potential treatments. PLoS One 13, e0190615.

[13] Saito E, Nakamoto BK, Mendez MF, Mehta B, McMurtray A (2014) Cost effective community based dementia screening: A markov model simulation. Int J Alzheimers Dis 2014, 103138

[14] Standfield LB, Comans T, Scuffham P (2018) A simulation of dementia epidemiology and resource use in Australia. Aust N Z J Public Health 42, 291-295.

[15] Green C, Handels R, Gustavsson A, Wimo A, Winblad B, Sköldunger A, Jönsson L (2019) Assessing costeffectiveness of early intervention in Alzheimer's disease: An open-source modeling framework. Alzheimers Dement 15, 1309-1321.

[16] Mar J, Soto-Gordoa M, Arrospide A, Moreno-Izco F, Martínez-Lage P (2015) Fitting the epidemiology and neu- 
ropathology of the early stages of Alzheimer's disease to prevent dementia. Alzheimers Res Ther 7, 1-8.

[17] Kansal AR, Tafazzoli A, Ishak KJ, Krotneva S, Collaboration A (2018) Alzheimer's disease Archimedes condition-event simulator: Development and validation. Alzheimers Dement (N Y) 4, 76-88.

[18] Jutkowitz E, Kane RL, Gaugler JE, MacLehose RF, Dowd B, Kuntz KM (2017) Societal and family lifetime cost of dementia: Implications for policy. J Am Geriatr Soc 65 , 2169-2175.

[19] Perneczky R, Wagenpfeil S, Komossa K, Grimmer T, Diehl J, Kurz A (2006) Mapping scores onto stages: Mini-Mental State Examination and Clinical Dementia Rating. Am J Geriatr Psychiatry 14, 139-144.
[20] Srishyla D, Adam H, Shah K, Wu T, King T, Summers RL (2020) The association of Mini-Mental State Examination (MMSE) with Clinical Dementia Rating (CDR). Public Health Review 3, 1-7.

[21] Dufouil C, Beiser A, Chêne G, Seshadri S (2018) Are trends in dementia incidence associated with compression in morbidity? Evidence from the Framingham Heart Study. J Gerontol B Psychol Sci Soc Sci 73(Suppl 1), S65-S72. 